Letros, Lima 48 (88-82): 30-45, 1985.

\title{
Un fresco de Pérez de Alesio en la capilla Sistina
}

FRANCISCO STASTNY

Entre los murales pintados por algunos de los más célebres artis. tas del Renacimiento que decoran la Capilla Sistina se encuentran dos composiciones, que por ser obras de artistas menores del siglo XVI, no han merecido estudios muy detallados acerca de su origen y de la fecha en que fueron ejecutados. Representan la Resurrección de Cristo y el tema más desusado de la Disputa del Arcángel Miguel con el Demonio por el cuerpo de Moijés. Ambos están ubicados enfrentc del Juicio Final de Miguel Angel, en la pared de entrada a la Capilla, y constituyen las últimas escenas de los ciclos de la Vida de Cristo y de la Vida de Moisés que llegan a su término en ese lugar (1).

Estas pinturas han sido motivo de considerable confusión en los escritos de autores antiguos y modernos.-Sus presencia en lugar tan notorio ha sido eloorigenude diversaso hipótesjs infundadas (2). Los primeros frescos pintados en esa pared ( $y$ ahora perdidos) fueron atribuidos diversamente a Ghirlandajo, Salviati y Miguel Angel (3), mien-

(1) Para una descripción en detalle de estos frescos, véase L. D. Ettlinger, The Sistine Chapel before Michelangelo. Religious imagery and Papal primacy. Oxford, 1965. Para la historia de la Capilla, E. Steinmann, Die ISixtinishe Kapelle, Munich, 1901-1905; R. Salvini, E. Camosasca, L. Ragghienti, La Cappella Sistino in Vaticano, Milán, 1965.

(2) El nombre de Miguel Angel ha sido mencionado incorrectomente en relación a estos frescos por F. Preciado de la Vega, Carta a G. B. Ponfredi (1765) en G. G. Bottari, Raccolta di lettere sulla Pittura, Scultura ed Architettura, Roma, 1754-1773, VI (1768); L. Lanzi, Storia pittorica della Italia, 5ta. ed. Florencia, 1945, II, 265; C. Gambo, Michel Ange et son école, Novara, 1948. 33.

(3) Las referencias a Salviati se encuentran en G. P. Chattard, Nuova descrizione del Vaticano, Roma. 1762-67, II, 36; F. Cancellieri, Descrixione delle Cappelle Pontificie e Cordinalizie, Romo, 1788-90, 11, 21; G. Mancini, Vita di Luco Signorelli, Florencia, 1903,47; M. Solmi, Luco Signorelli, Novara, 1953, 47; H. Noe, Carel van Mander en Italie, La Hayo, 1954, 126, 317; E. Pistolesi, Descrizione di Roma e suoi contorni, Roma, 1846, 598; A. Nibby, Itinerario de Roma e suoi dintorni, Roma, 1877, 400-02. Ambos frescns fueron atribuidos a Ghirlandaio por $H$. Voss, Die Malerei des Spätrenaissance in Rom und Florenz, Berlin 1920, I, 571. 
tras que ambas composiciones conservadas han sido asignadas frecuentemente al artista italo-español Mateo Pérez de Alesio (4). Muchos escritores han descrito el tema representado, erróneamente, como la Tentación de San Antonio o como la Caida de los Angeles rebel des (5). Y por añadidura, la falta de evidencia documental ha dejado en duda la fecha exacta de su ejecución.

El propósito de este artículo es revisar la historia de la decoración de la pared oriental de la Capilla Sistina, a fin de establecer con más precisión la fecha en que los frescos pudieron haber sido pintados $y$, así, contribuir a explicar su presencia en la Capilla.

A pesar de lo que pueda parecer a primera vista, el interés que cste tema posee para la historia del arte peruano es considerable. El autor de uno de los murales que se se van a examinar, fue Mateo Pérez de Alesio o Matteo da Lecce, como lo llamaron los italianos, quien fue uno de los fundadores; junto con Bernardo Bitti, de la escuela de pintura virreinal en el Perú (5?). De modo que los datos que se van a precisar a continuación servirán, por un lado, para disipar el mito de la relación personal de Pérez de Alesio con Miguel Angel, abundantemente repetido en la literatura peruana sobre el pintor, y por otro, para explicar mejor el ambiente artístico y religioso del cual provino Mateo de Alesio antes de su llegada al Nuevo Mundo.

La primera decoración mural por el grupo de pintores "detti antichi moderni" (6), fue encomendada por Sisto IV el 27 de octubre de 1481. Domenico Ghirlandajo contribuyó en ese proyecto con una $R e$ surrección de Cristo, en la banda izquierda de la pared del lado oriental. La escena equivalente en el ciclo del Antiguo Testamento, la $D e-$ fensa del Cuerpo de Moisés, fue pintada por Luca Signorelli en el costado derecho del mismo muro, poco después de octubre de 1482 (7).

Las composiciones de Ghirlandajoly Signorelli sufrieron graves

(4) Por ejemplo, G. Celio, Delfj nomi dell'artefici delle pitture che sono in alcune chiese, facciote e palazzi di Roma. Nápoles, 1638, 102; F. Titti, Ammaestramento utile e eúrioso di pitturo, scultura et architetturo nelle chiese di Roma. Roma, 1686, 409; F. Preclado de la Vega, Loc. cit.; G. Roisecco, Roma antica e moderna o sio nouva descrizione della Cíta di Roma, 1745, I, 70; G. Vasi, ltinerario istruttivo... per rifrovare... le Antiche o Moderne mognificenze di Róma, Roma, 1777, 500 . L. D. Ettlinger, Op. eit., $14 \mathrm{ff.}$

(5). Este érror émpezí con G. Baglione: Le vite de pittori, scultori, et architetti dal Pontificoto di Gregorio XIII... Roma, 1642, 32; véase también: J. Meyer, Allgemeines Kunstler-Lexikon, Leipzig, 1932, XXV1, 409-10; M. S. Sorio, La pintura del siglo XVI en Sudamérica, Buenos Aires, 1956, 74; Idem. "Pintores itolianos en Sud América"; Saggi e Memorio di Storía dell'Arte, 4, 1965, 127; D. Angulo, Pintura del Renacimiento, Madrid, 1954, 266.

(5o) Ver nota 9.

(6) La terminología histórica de Vosart fue aplicada a la Capilla Sixtina por G. Celio, loc cit. "...le pitture intorno sotto la cornice erano di mano delli primi artefici, ... detti antichi moderni, perche non sono li antichi Greci, ne ii modemi, che sono dopo Pieto Perugino, ...".".

(7) G. Vosari, Le vite de' piu eccelenti pittori, scultori ed architetti, (1568), ed. G. Milanesi, Florencia, 1878-1885, III, 259, 702, simplificando el título, Vo. sari llamó a la pinturo de Signorelłi: Lo Muerte de Moisás. 
daños cuando el pórtico que se halla en la misma pared tuvo que ser reconstruido $(8)$. Y en una fecha muy posterior los dos frescos actuales fueron ejecutados por Mateo Pérez de Alesio (9) (la defensa del Cuerpo de Moisés) y por Henri Van den Broeck (10) (la Resurrecición (11).

Ia destrucción del fresco de Signorelli nos priva de un elemento de comparación con su célebre obra en Orvieto, la cual fue probablemente anticipada en su vigoroso dinamismo por el mural de la Capilla Sistina (12). La gracia y la energía que debió exhibir la composición perdida, probableriente está reflejada en el dibujo de Signorelli de un Arcángel con una espada desenvainada de la colección del Uffizi (13).

(8) Ver la nota 7.

(9) Mateo Pérez de Alesio (llamado Matteo da Lecce) nació hacia 1547 en Alezio, Puglia. En 1568-69 trabajó en la Villa d'Este, Tivoli, y permaneció en Roma hasta 1576, recibiendo algunos encargos importantes en la Capilla Sixtina y en el Oratorio del Gonfalone. De 1576 a 1581 vivió en Malta. Regresó a Roma de 1581 a 1583. Se inscribió en la Academia de San Luca (1573) y en la Congregczione dei Virtuosi (1583). Viajó a Sevilla en 1583 y desde 1590 está claramente documentado en Lima, Perú, donde falleció entre 1607 y 1616. Véase C. van Mander, Het Schilderboeck, Harlem, 1604, 193v-194; G. Mancini,: Considerazioni sullo pittura, Roma, 1956, I, 222, II, ns. 866-869; G. Baglione, loc. cit.; J. A. Ceán Bermúdez, Diccionario de los Bellas Artes en Espoño, Madrid, 1800, IX, 75-78; J. Mayer, loc. cit.; U. Thieme, F. Becker, loc. cit. Pora la fase Hispono-Americana de su vida, véase R. Vargas Ugarte, Ensoyo de un Diccionario de Artífices de lo América Meridional, Burgos, 1968, 127 130; F. Stostny "Pérez de Alesio y lo Pintura del Siglo XVI", Anales del Instituto de Arte Americano y de Investigociones Estéticas, 22, (Búenos Aires), 1969, 9-46; Idem. M. Pérez de Alesio and the development of Peruvion Colonial Pointing, Tulane University, Symposia sobre Arte Latinoamericano, New Orleans, 1972; M. Soria, loc. cit.: J.Mesa-Gisbert, El pintor Motco Pérer de Alesio, LG Paz, 1972; J. Bernales B., "Mateo Pérez de Alesio", Archivo Hiopolense, LVI, $171-173,1973,221 \mathrm{f}$.

(10) Hendrick van den Broeck (llamado Arrigo Fiammingo. Arrigo Poludano. Henrico de Mulines, etc.) nació en Malines hacio 1530. Estudió con Frans Floris y viaió a Italia donde visitó Florencia (hacia 1550), Orvieto (1561), Perugia (1562) y Roma (desde 1565). Falleció entre 1592 y 1605 . Véase L. Guicciardini, Descrittione di Tutti i Poesi Bassi, Antwerpen, 1567, 99, 101: G. Baglione, op. cit., 77; U. Thieme, F. Becker, op. cit., V.; C. J. Hoogewerff, Nederlondsche Schilders in Itolie in de XVIe Eeuw, Utrecht 1912, 162; W. Bombe. "Arrigo Fiommingo", Revue Belge d'Archéologie et d'Histoire de I'Art, 5 , 1935, 231-144; L. van Puyvelde, Lo peinture flammande ò Rome, Brusselas, $1950,45-52 ; \dot{H}$. Nöe, op. cit. 125, ns. 1-3. 40,45 .

(11) A. Taja, Descrizione del Polazzo Apostolico Voticono, Roma, 1750,

(12) La contienda entre las fuerzas del bien y del mal representada en el fresco de Signorelli, probablemente estaba inspirada en Dante, como lo estuvo luegó su trabajo en Orvieto. Este aspecto de los frescos de Orvieto ha sido estudiado por A. Venturi, Luco Signorelli interprete di Dante, Florencia, 1921,24 ; véose también $G$. Mancini, op. cit., (1903) y $M$. Salmi, op. cit., 12, 13, 70 , quien sugirió que la fuente paro el fresco del Vaticano debió ser el Purgatorio, $V$, donde el poeta describe la lucha de un ángel con un demonio por el alma de Buonconte.

(13) Gabinetto de Disenni e Stampe, Uffizi, N. 50 E. Carboncillo $250 \times$ $170 \mathrm{~mm}$. Véase Mostra de L. Signorelli. Cortona y Florencia, 1953, 141 , No. 74. 


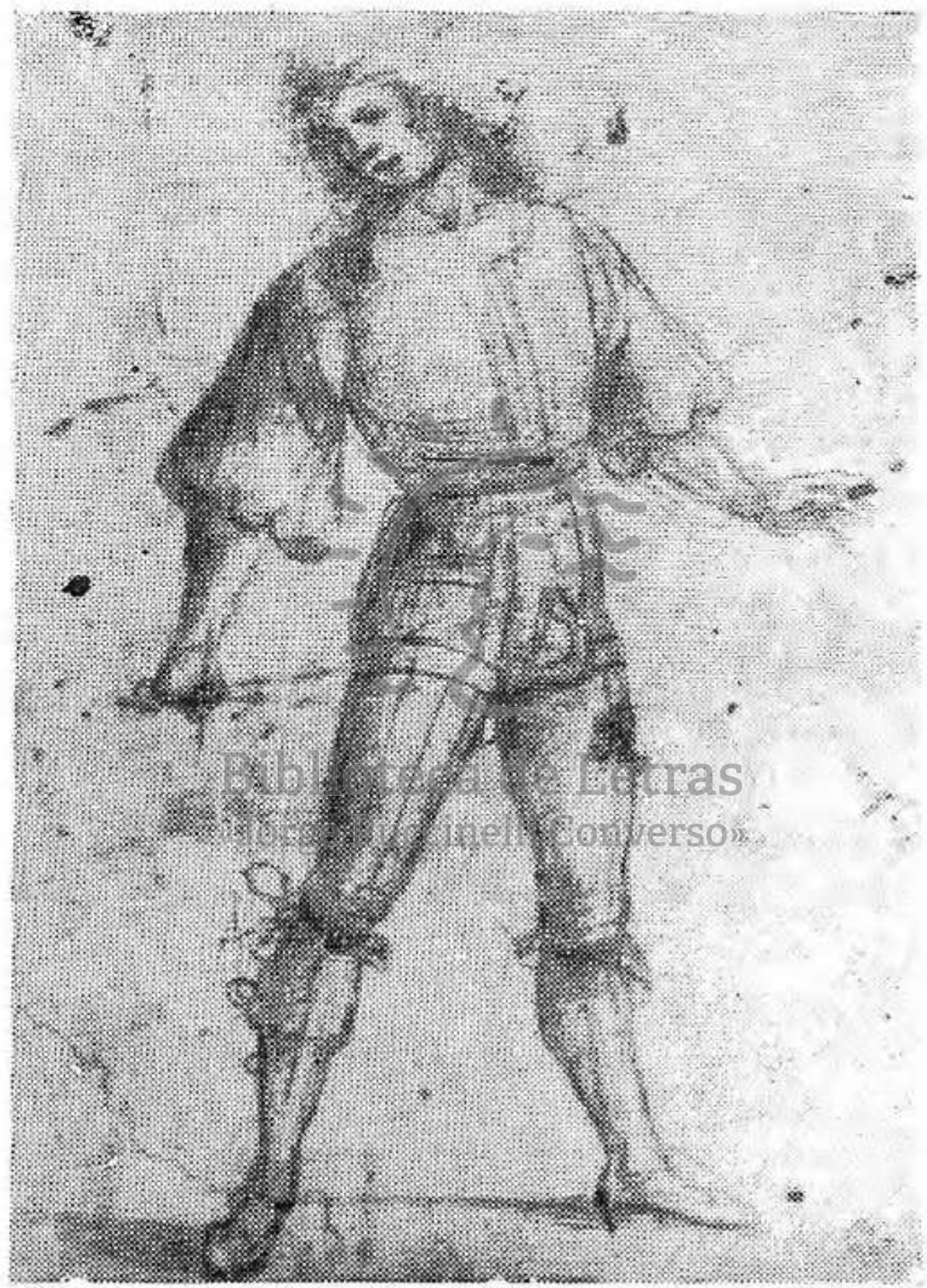

Foto No. 3. "Arcóngel con una espada desenvainoda", dibujo de Lucas Signorelli. Colección del Uffizi. 


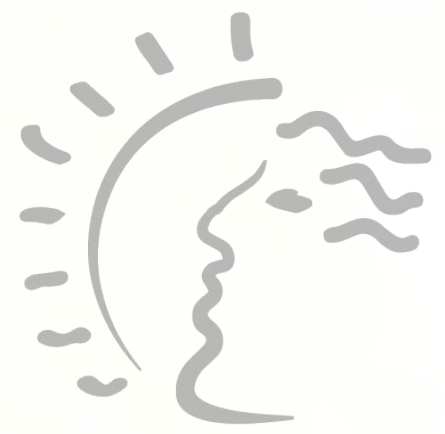

Biblioteca de Letras "Jorge Puccinelli Converso» 
El estilo temprano de ese diseño (14) y la posición de la figura, similar en el gesto del brazo extendido al Arcángel en el fresco de Alesio, son suficientes razones para creer que ese carboncillo pueda ser un estudio para la figura central del mural perdido (15).

El siguiente acontecimiento en la historia de la decoración de aquella pared, tuvo lugar cuando el dintel de la puerta que da acceso a la Capilla se quebró y cayó en vísperas del 25 de diciembre de 1522 (16). La Resurrección de Ghirlandajo, informa Vasari, fue "guasto en maggior parte" durante los trabajos que se tuvieron que emprender para reemplazar "il architrave che revino" (17). La información no es repetida por Varasi en la Vida de Signorelli, pero dada la posición del fresco debe haber sufrido la misma suerte (18).

Por lo común no se menciona que el interés de Clemente VII por obtener que Miguel Angel pintara nuevos frescos en la Capilla Sistina se debió sobre todo al estado ruinoso de las dos escenas de la pared de entrada. El deseo del Papa de sustituir los frescos dañados por una gran Caída de los Angeles rebelde.;, y de disponer un Juicio Final en el muro del altar, fue comunicado al artista, quien, según Vasari, preparó varios estudios para ambas composiciones (19). Cuando Pablo III insistió en el pedido, Miguel Angel se enfrascó en el proyecto más osado, el Juicio Final (20). En jambio, llegó a tomar en considera-

(14) Estó relacionado con Perugino, quien también participó en la decoración de la Capilla, véase M. Marangoni, I disegni della R. Galleria degli Uffizi, Florencia, 1912-19́21 como aparece señalado por M. Moriando en Mostra, loc. cit.

(15) El personaje parece rechazar a un enemigo con un fuerte impulso hacio la parte baja de la esquina izquierda. Este movimiento coincide con la tendencio de la composición adoptada en la Vida de Moisés, que va de derecha a izquierda. Un personaje "con שy movimientol de cabezo Muy similar aparece en la esceno de Moisés señala a su sucesor Josué en el fresco de Signorelli. En el dibujo no se percibe ninguna relación con los Arcángeles armados de Orvieto.

(16) El dintel cayó cuando el Papa estaba por entrar en la Capila y mató a dos soldados de su séquito. El accidente es narrado por P. Giovio, Delle istoric del suo tempo, (1550-52), Venecio, 1608, señalado por R. Salvini, E. Camesascs, L. Ragghianti, Op. cit., I, 133, 275.

(17) Los frescos se destruyeron por la restauración realizada fara reforzar la entrada, más que por la verdadera caída del dintel. G. Vasari, op. cii,. III, 259: "La Resurrezione ... e guasta la maggior parte, per essere ella sopra lá porta rispetto all'avervisi cvuto a rimetter un architrave che rovinó". D. Redig de Compos, I Palozzi Yaticani, Bologna, 1967, 68.

(18) M. Solmi, op. cit., 70.

(19) "... voleva Clemente che... dove e l'altare, vi si dipignesse il Guidizio Universale... e sopro la porta principole gli aveva ordinato che vi facesse, quando per le sua sobertia Lucifero fu dal cielo cacciato....; delle quali invenzione molti anni innanzi s'e trovato che aveva fatto schizzo Michelongelo e vari disegni...". G. Vasari. op. cit., VII. La información que añade Vasari, de que uno de los dibujos fue copiodo en Trinità dei Monti por "un pittore siciliano" que habio sido ayudante de Miguel Angel, llevo a la identificación errado del siciliano con Muteo de Alesio (probablemente sobre la base de qua Von Mander lo llama siciliano), véase C. Gombo, loc. eit.

(20) Ch. Tolnay, Mi hal Ange, Poris, 1951, 72, 73, 250. 
ción los dos frescos de la pared opuesta (21) Sin duda, las razones de orden iconográfico fueron cruciales en la determinación de no reemplazar la Restureción y la Defensa del Cuerpo de Moisés. La impor. tancia del primer tema como culminación triunfal del ciclo de la vida del Salvador, es evidente; en cambio la segunda escena tiene un definido significado simbólico en relación a la autoridad papal, $y$ por ese motivo debió haber sido particularmente apreciada por los pontífices de los difíciles años del siglo XVI (22).

Extrañamente, los murales de la pared oriental permanecieron en estado ruinoso durante casi medio siglo, después de la reconstrucción del dintel alrededor de 1522 . El emblema de Pío IV encima de la puerta recuerda que durante su pontificado (1559-1565) fue renovado el revestimiento de mármol del pórtico; no obstante los frescos no fueron restaurados en esa época (23). En 1561 el Papa le encargó a Francesco Salviati parte de la decoración de la Sala Regia (24) y algunos autores han asumido equivocadamente que también pintó la Defensa

(21) El grupo de dibujos de Miguel Angel sobre lo Resurrección, de alrededor de 1530, no estón relocionados con el proyecto de la Capilla Sixtina, como fusro sugerido por $C$. Gamba, Pittura di Michelangelo, Novara, 1945, XXVIII, XXXII; Idem, op. cit., 33. Tampoco hay ningún dibujo asociado a la obra dé Alesio. Los dibujos de la Resurrecion son en su moyor porte "dibujos de presentoción", véase J. Wilde, lfalian drawings in the British Museum. Michefangelo and his studio, Londres, 1953, Nos. 52 y 54, 90. Paro la discusión de este osunto véase tambièn A. E. Popham $y$ J. Wilde, The Italian drawings of the $X V$ and $X Y I$ centuries in the collection of his Majesty the King of Windsar Castle, Londres, 1949, No. 427, 251.

(22) Sixto IV vio en ese temo la prueba de que incluso ei Demonio debe obederer los mandatos de Dios. Por extension. el Popa debía tener poder ilimitado, tnnto en los asuntos espirituales como temporales. El tema de la autoridad napa! fue de gran greocupación poro isixio'Sy"y se volvió más actual en el Singlo XV! camo punto de debote entre la Iglesia y los Reformistas. Aunque no hoy ninguna otra representación de eso escena en gran escalo, se comprende que los pontifices de épocos posteriores decidieran conservarla. Tombién fue difundido a fines del Siglo $X V I$ en uno estampa diseñoao por Martin de Vos y graboda por Wierix. Véase F. dello Rovere (Sixto IV). De Potentia Dei, Roma, 1471 , 10v. citado por L. D. Ettlinger, op. cit., 74, 75; E. Steinmonn, op. cit., I, 516, n. 2.

9. En la Edad Media fue tratado por J. da Voragine, Legenda Aurea, Ed. Th. Grasese, Bratislovia, 1890, 642 (citado por Ettlinger, loc. cit.), con un significado difarents: La intervención de Son Miguel, tiene el propósito de impedir que los lsraelitas adoren el cuerpo da Moisćs. Esta interpretación también la da A. Tojo, Op. cit., 40.

(23) El hecho de que Vosari no mencione ningún cambio en la condición del fresce de Ghirlondaio en lo segunda edición de Yite es una prueba de que en 1568 , durante el pontificado de Pío $V$, la pored aun estaba malograda, véa. se G. Yssari, op cit., III. 259; Ed. Steinmann, op. cit., I, 166, No. 2.

(24) Salviati pintó Barbarroja y Alejandra III en lá Sala Regia, a la espoldo de la pared de la Capilla Sixtina, donde está colocado el emblema de Pio IV. Pero al poco tiempo Salviati sc enerristó con el Popa, no hobiendo ningu. no razón para creer que pintara también en lo Capilla, especialmente, ya que Vusari, su amigo y compagno, no hace ninguna mención al respecto, véose $H$. voss, op. cit., 1, 256 y referencia $3 ; G$. Moncini, op. eit., (1956), 1, 231. 


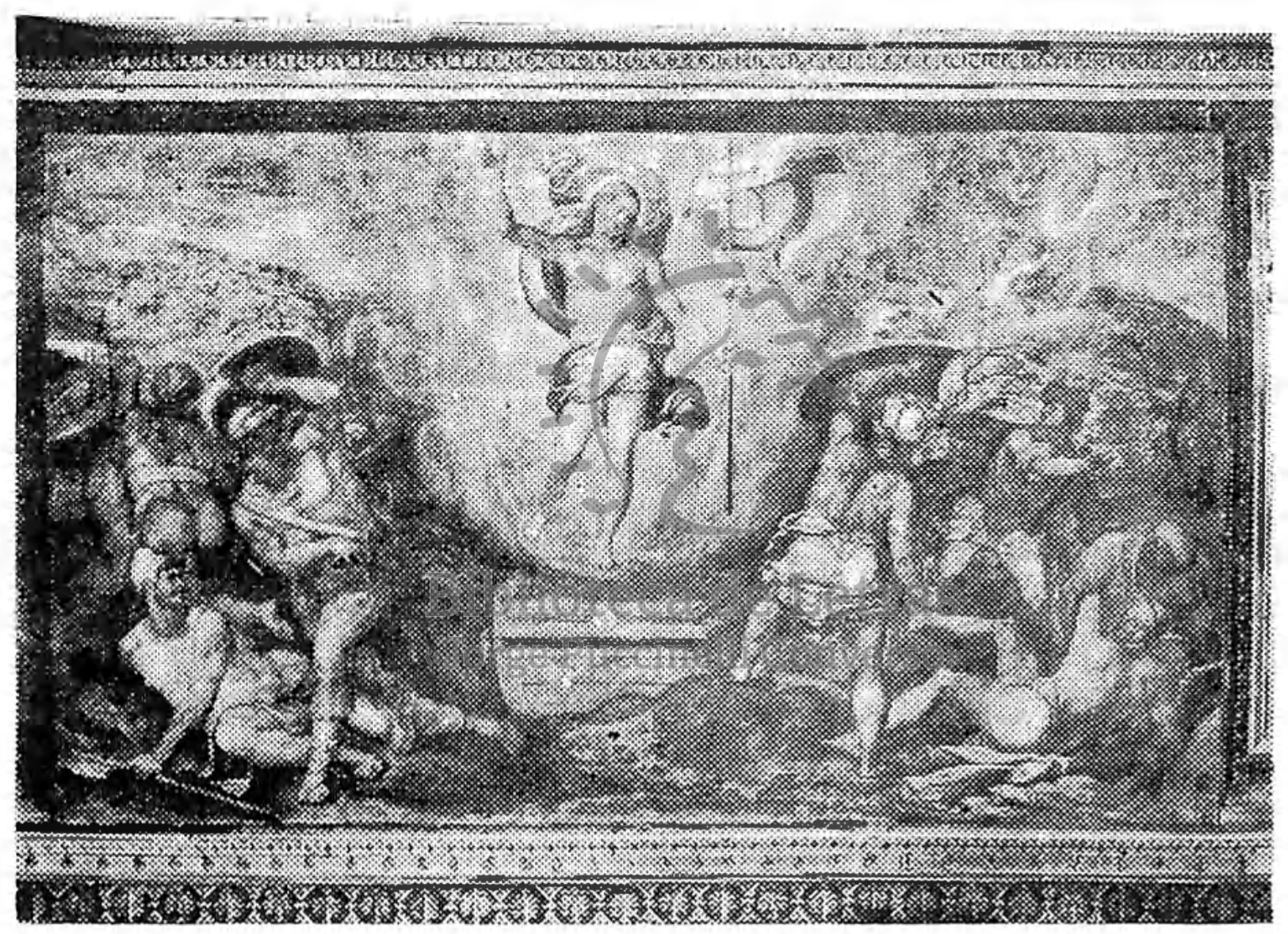

ROMA - La Resurrección - Salviati - Capilla Sistina - Vaticano. Anderson. 


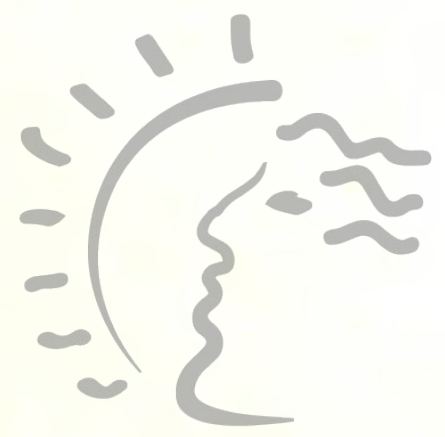

Biblioteca de Letras "Jorge Puccinelli Converso» 
del cuerpo de Moisés. la cual habría sido restaurada, posteriormente, por Mateo da Lecce (25).

La Capilla tuvo que sufrir aún mayores daños antes de que se considerara la restauración de los frescos. En octubre de 1565 se descubrieron algunas grietas amenazadoras en la pared del lado norte y del cielorraso que ocasionaron serias inquietudes por la seguridad de la estructura. No se podía seguir oficiando misa en la Capilla y se tuvo que desalojar todos los bienes de la Sacristía. Finalmente, entre julio de 1568 y actubre de 1569 la construcción fue íntegramente reparada por Pío V (26) con gran costo.

Una vez que los muros fueron consolidados, el Cardenal Rusticucci prosiguió, en acatamiento de las instrucciones del Papa, con la refacción de las pinturas. Según Bottari, llamó a Girolamo de Fano para restaurar los frescos de Miguel Angel y para terminar de cubrir los desnudos que Daniel de Volterra había dejado sin velar en el Gilldizio. De Fano falleció poco después y su asistente Domenico Carnevale quedó a cargo de la mayor parte de la obra (27). Un pago a Carnevale está documentado en diciembre de 1569 (28), pero las labores debieron de proseguir mucho tiempo después.

La retribución of recida a Carnavale es el último documento conocido acerca de esta fase de la historia de la Capilla. No se han identificado contratos o recibos de pagos para las obras de Alesio o de Arrigo Fiammingo. Es por eso que la atribución de los frescos y su datación, en los términos aceptados hasta ahora, han sido derivadas de fuentes secundarias.

(25) A. Taja, op. cir., 40,45 repite a Baglione diciendo que Alesio se propuso imitar el estilo de Salviati. G. P. Chattard, loc. cit., no comprendió la información y escribió inexactametne que el fresco primeron fue pintado por Salviati y luego rehecho por Alesio en tiempo de Gregorio XIII, después de la roturo del "arquitrabe" (lo que habría sucedido asi en una fecha completamente difs. rente), véase $E$. Steinmann, loc. cit.

(26) E. Steinmann, op. cit., II, 779-783; R. Salvini, op. cit., I, 124; A. Bertolotti, Artisti modonesi, parmesi e della lunigiana in Roma nei secoli XVI, XVII e XVIII, Modena, 1882, 22. Pio IV tuvo que trasladarse de sus apartamentos a un lugar más seguro y luego de su muerte, durante la realizacićn del conclave, se dio un permiso especial a los arquitectos para que entraran a revisar la condición de la Capilla. Mientras tanto el Papa tuvo que celebrar misa en la iglesia de San Pedro. Están registrados los pagos de 5000 cscudos a Giacinto Barrozzi por el trabajo en la Capilla. Pirro Ligorio realizó un dibujo (Biblioteca Bodleian) sobre la forma cómo se podría reparar la estructura, pero no se ha podido probar su participación en el trabajo, véase D. Steinmann, op. cit., I, 152, fig. 72 .

(27) La principal contribucićn de Carnevale fue en el Sacrificio de Noś donde se había desprendido un gran pedazo de intonaco, según F. Forceroli. La información sobre ambos pintores fue publicada por $G$. Bottari, Vita de Mickelangelo Buonarroti scritta da Giorgio Vasari, Roma, 1760, 176, n. 10, quien se re fiere a un manuscrito acerca de "Uomini illustri Modanesi", por Francesco For celoli y a Vedriani: Raccolta de'pittori 3.c. Modanesi., 99; véase E. Steinmann, op. cit., 11,783, n. 6 .

(28) L. Pastor, Storia dei papi della fine del Medio-evo, Roma, 1925 1934, VIII, 86 n. 3. 
Que Mateo Pérez de Alesio pintara la defensa del cuerpo de Moisés está confirmado por el testimonio de primera mano de dos autores: por Carel van Mander, quien estuvo presente en Roma entre 1574 y 1576 y lo describió correctamente en el Schilderboech, agregando la extraña información de que "sin embargo el Angel... es la obra de Guidonio" (29); y por Francisco Pacheco, quien siendo aún un jovenzuelo conoció a Alesio en Sevilla y años más tarde recordó claramente el dibujo de la "Muerte de Moisés". Fue el mejor dibujo de Alesio, escribió con admiración, "porque había sido pintado delante del Juicio de Miguel Angel y asumió su gran manera". La veracidad de esta noticia, agregó Pacheco (30), fue probada "por la información otorgada por algunas personas que habían estado en Roma y habían visto la pintura" (31).

Henrico Fiammingo es mencionado por primera vez como autor de la Resurrección en un manuscrito anónimo de ca. 1600 (32) y el dato fue repetido correctamente por Baglione (33). Por añadidura, la obra está claramente firmada con el monograma del artista, sobre el sarcófago allí representado de modo que no queda ninguna duda sobre quién pueda ser el autor (34).

Para un ojo moderno, la unidad del esquema formal de los frescos del siglo XV es interrumpida por la intrusión de las obras de Van den Broeck y de Alesio. I.a escala de sus figuras y el principio de su construcción, basado mayormente en un concepto restringido de la figura

(29) C. van Mander, op. cit., 193 v-194; M. Vaes, Le séjour de Carel van Mander en Italie, 1573-1577, Homenaje a Dom U. Belière, Bruselas, 1931, 228, n. 1, 237, n. 2. El pintor Guidonio fue identificado de primera intención por $M$. Vaes, como Galeazzo Ghidoni, natural de Cremono en 1598. Si tenía 2025 años cuando conocić a Van Mander, quiere decir que falleció a la edad de 43-48 años, ciertamente ge no d una edád temprana." Véase M. Vaes, "Appunti di C. van Mander su vari pittori italiani suoi contemporanei", Roma, IX, Mayo-Sept., 1931; U. Thieme, F. Becker, op. cit., XIII, 547. Sé dabería buscar otros artistas ccn el nombre cristiano de Guido o Guidonio para ocompoñar la información de Van Mander: por ejemplo, un Guidonio Guelfi del Borgo estaba matriculado en la Academia de San Luca, alrededor de la mismo época que $\mathrm{H}$. van den Broeck, hacia 1580. Véase Libro di Introito, 1553-1633, II, f. 9a. Archivo Storico della Accademia di San Luca. Guidonio Gueufi también colaboró con Alesio en marzo de 1582, pintondo un emblema de Gregorio Xill en la decoración del Panteón fora las fiestas de la Congregazione dei Virtuosi; véase $\mathrm{H}$. Waga: "Vita nota e ionota dei Virtuosi al Pantheon", L'Urbe, 5, 1967, 2.

(30) Lo participeción de Alesio en la Capilla Sixtina, despertó él escepticismo de los artistas sevillanos. Al famoso escultor Jerónimo Hernández (ca. $1540-1586$ ) se le recuerda diciendo sarcásticamente que si el dibujo fuero realmente de Alesio se hario gustosamente su discípulo, véose F. Pacheco, Aite de la pintura, su antiguedad y grandeza, Sevilla, (1649), edición 1866, 11, 10-11.

(31) Extrañamerite, Baglione (Op. cit., 32) que usualmente es exacto, comete el error de asignar ambos frescos, con títulos equivocodos, a Alesio, véase lo nota 5 .

(32) Eleucl:us, f. 25, citado por $\overline{\text { J. }}$ I. Hoogewerff, Beschieden en Italie ombrent Nederlandsche Kunstenaars en Galeerden, Lo Hayo, 1917, 111, 140.

(33) G. Baglione, op. cit., 77.

(34) El monograma está compuesto por las letras HVDB, véose G. I. Hoo gewerff, op. cit., 1921, 162. 


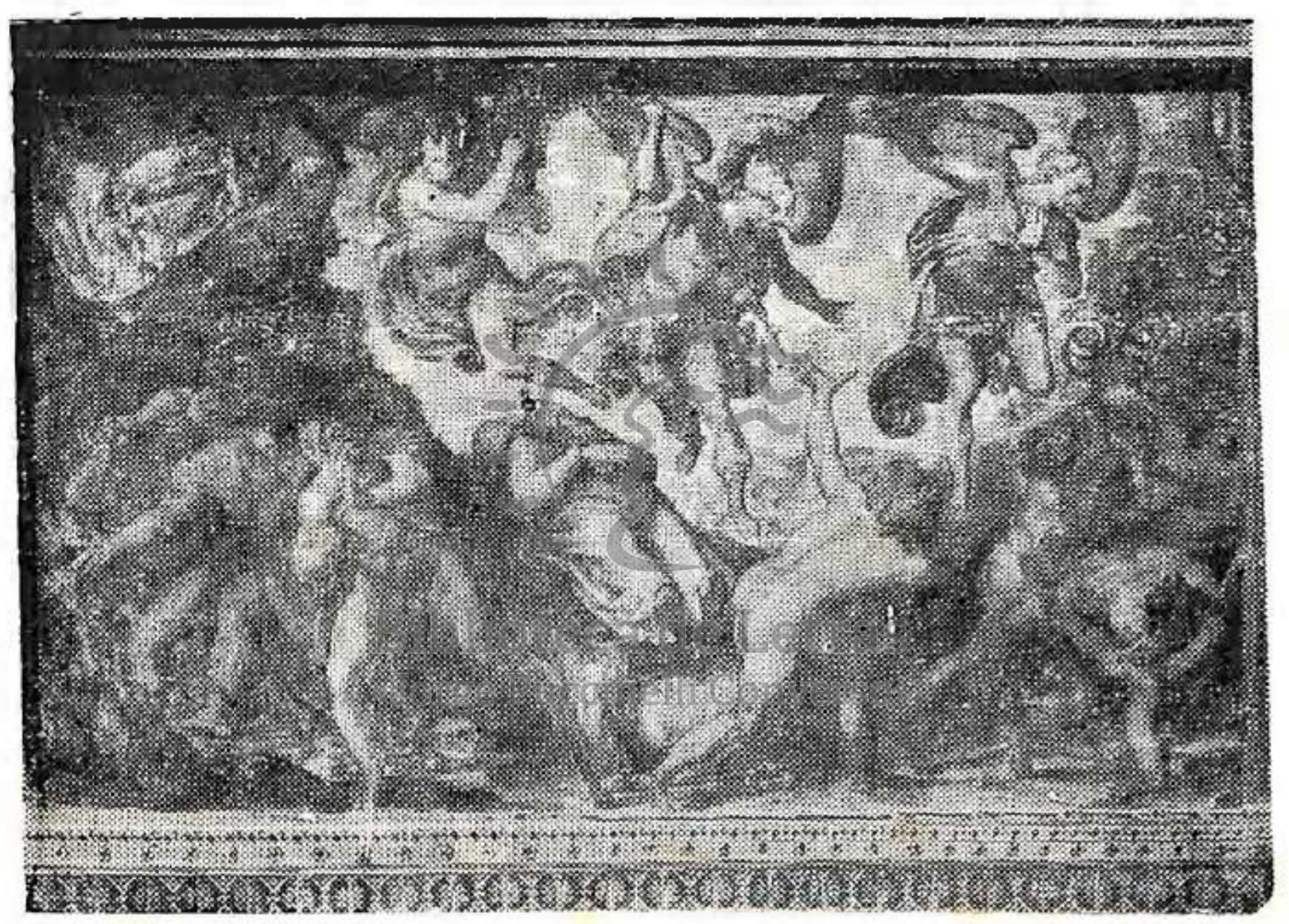

ROMA - La defensa del cuerpo de Moisés - Solviati - Copilla Sistina - Vaticano. Anderson. 


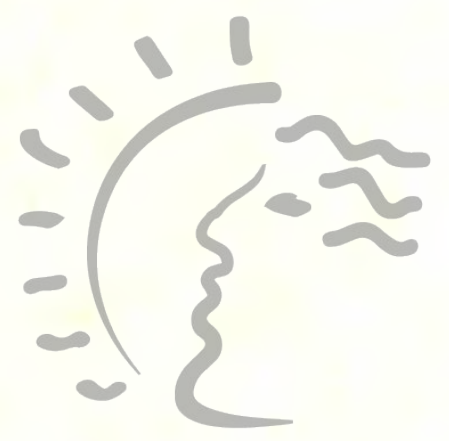

Biblioteca de Letras "Jorge Puccinelli Converso» 
serpentinita, son totalmente distintos a los de las pinturas más antiguas; así como lo es el tratamiento del espacio, el cual se vuelve denso, sobrecargado con figuras humanas de grandes dimensiones, mientras que las escenas más antiguas están colocadas delante de un mundo armonioso de perspectivas perfectamente ordenadas.

No obstante, un examen más detenido revelará que los frescos del siglo XVI también fueron concebidos coherentemente sobre la base de una similar estructura formal y de que, dentro de los límites de su propia idiosincracia estilística, fueron adaptados deliberadamente al esquema del siglo anterior. Ambas obras tienen una hilera de figuras en el primer plano, que repite la distribución empleada en las pinturas de los antiguos maestros. Están construidos según el principio de una composición radial que se desarrolla alrededor de un eje vertical en un movimiento de expansión semi-circular; y en los dos murales el espacio está dividido en tres zonas según un esquema en forma de " $V$ ".

El movimiento que se percibe en el progreso de los dos ciclos, que avanzan en sentidos opuestos, también es respetado, ya que cada uno de ellos el evento final de la historia está representado como una pequeña escena en el fondo, una frente a la otra: La Ascención de Cristc, en el ángulo superior derecho de la Resurrección, y El Entierro de Mo:sés, en el ángulo superior izquierdo del otro mural.

A pesar de que en ambos frescos se evidencia un tratamiento formal similar, por lo común se ha asumido que la obra de Van den Broeck representa una versión más fiel del mural antiguo que la de Alesio. Esta idea es sostenida, sobre todo, por la mesura en la concepción de la figura de Cristo (35) y por el mayor énfasis de la composición en su movimiento hacia el lado derecho, en concordancia con el ritmo del ciclo original. En la obra de Alesio a pesar de que los ángeles crean al impresión de progresar de derecha a izquierda, la búsqueda de una contorsión serpentinata ha invertido el efecto y el movimiento con que está impulsada la lanza del Arcárgel Miguel en sentido contrario arroja las figuras más prominentes de los demonios de regreso hacia el ángulo derecho (36).

(35) La anatomía derecha de Cristo con mínima indicación de contrapposto, revela que Van den Broeck siguió fielmente (aunque en forma literal) el mo. delo del Siglo XV. Unicamente la postura mós desplazada de los miembros indica que es uina obra posterior. La necesidad de armonizar con un modelo arcaico es aún mós perceptible si se compara con la contemporánes Expulsión dei Paraíso de Van den Broeck (S. María degli Angeli, Roma), donde la enérgica figura de Adón estó tomada directamente del Juicio 'Final de' Miguel Angel, véase T. H. Fokker, Werke Niederländische Meister in der Kirchen Italien. La Haya, 1931, 126; Dipinti Fiamminghi di Collezioni Romane, Palazzo Barberini, Roma, 1966,16 , fig. 17.

(36) La manera en que el Arcángel Miguel rompe el esquema establecido de la composición, parece confirmar el informe de Van Mander acerca de la intervención de un pintor diferente llamado Guidonio. Quien quiera que fuese, su participación no puede entenderse como el trabajo de un simple ayudante, como sugiere $M$. Noë, op. cit., 126. 
En ambos frescos los modelos dominantes han sido las densas estructuras de las figuras que pueblan la Capilla Paolina y las obras tardías de Miguel Angel (37). La Resurección de Van den Broeck fue compuesta ignorando deliberadamente los dibujos de la década de 1530 del Buonarroti dedicados al mismo tema (38). La ausencia de todo vestigio de la grazia característica de los diseños más tempranos de Miguel Angel es el resultado de la búsqueda consciente de un idioma más sobrio para la pintura religiosa. Los artistas romanos de la Contramaniera estuvieron inspirados por el desarrollo tardío del genial florentino en su aspiración por una nueva modalidad artística más grave y circunspecta. Inclusive el respeto por composiciones de tiempos anteriores, como en el caso aquí estudiado, coincide con esa tendencia de los años en torno a 1570 , que se desarrolló en una atmósfera dominada por la piedad de Pío V y por una actitud "pasadista" como lá que florecía entonces en Roma y que irradiaba sobre todo del influyente Palacio Farnese. El importante lugar que ocupan los dos murales, condujo a que, a pesar de no ser sino obras de artistas limitados, estuviesen cuidadosamente afinados a las últimas exigencias ideológicas de sus días. En esc sentido reflejan con toda fidelidad la preocupación del Cinquecento tardío por emplear un vocabulario artístico capaz de evitar los excesos formales y la frialdad emocional de la Maniera (39).

Se ha asumido habitualmente que los frescos de Alesio y Van den Broeck fueron pintados durante el pontificado de Gregorio XIII, en algún momento entre 1573 y 1575 (40). Una antigua tradición, reiterada por la mayoría de los autores que se han ocupado del tema y probablemente originalda en cl caso de Pérez de Alesio en el propio artista, sustenta esa idea. El historiador sevillano Gonzalo Argote de "Jorge Puccinelli Converso"

(37) Los ángeles en la Defensa de Moisés son una excepción, ya que se oponen estilísticamente al resto de los personajes. Su elegancia ornamental se deriva de Taddeo Zuccari, quien utilizó un movimiento interrumpido similar con ei talle en torsión, en algunos de sus ángeles en vuelo o de personajes en acción. Taddeo pintó también en su Conversión de San Pablo, de S. Marcello al Corso, los mismos drapeados con espirales convencionales como se ven en el ángel del lado derecho, así como una versión célebre de la mano que señala (derivada de ia Copilla Paulina) y utilizado como modelo cara el Arcángel Miguel. Véase el dibujo de Taddeo El Angel advirtiendo a San José que huya a Egipto. (Kunsthalle, Hamburgo) o el Ecce Homo de S. Maria della Consolazione, Roma. De chí que el origen fundamental del estilo de Guidonio, resida en la tradición de Ratael y no de Miguel Angel, a diferencio del resto del fresco. Véase J. Gere, Taddeo Zuccaro, Londres, 1969, 158, Cot. 86, figs. 78, 72, 104.

(38) Para los reproducciones de los dibujos y la bibliografía véase $A$. $E$. Popham, J. Wilde, op. cit., 251 , No. 427, pl. 22; J. Wilde, op. cit., 90-91, Nos. 52-54; véase también la noto 21.

(39) Para el ambiente en Roma y la actitud retrospectiva neofeudal de los Fornese, véase F. Zeri, Pittura e Controriforma, Turin, 1957, 44 ss., y la nota 64. Para la relación del estilo de la Contramaniera con la Maniera y el clima religicso, véase S. J. Freedberg, Painting in Itoly, 1500 to 1600, Harmondsworih, 1970, 293-295.

(40) A. Nolfino. L'Oratoris del Gonfalone, Roma, 1964, 35; H. Noë, op. cit., 126 
Molina, lo calificó de "pintor de su Santidad" ( en una obra publicada en fecha tan temprana como 1588 . El cronista agustino Antonio de la Calancha, quien pudo haber conocido al artista en Lima, fue más lejos y lo describió como "aquel... raro pintor... que lo fue del Papa Gregorio Decimotercio". En Italia, Baglione mencionó a Alesio y a Van den Broeck entre los artistas que trabajan para Gregorio XII (41), y Taja fue el primero en decir explícitamente que los dos frescos fueron renovados por encargo de ese Papa. Desde entonces esa información se ha incorporado en los escritos modernos acerca de la Capilla Sistina (42).

Alguna vinculación con Gregorio XIII se ve confirmada, además, por la presencia del emblema del Papa - un dragón alado y sin colaen un lugar algo disimulado de la Defensa del Cuerpo de Moisés. Aparece en la tarja que decora el frente del sarcófago, en el contexto de una representación de la Zarza Ardiente (43).

La prueba parece incontrovertible. No obstante, las conclusiones aparentes a que ésta conduce son difíciles de aceptar. ¿Cómo es posible que dos pintores sin importancia hubiesen sido llamados en los tiempos de Gregorio XIII para una obra de tanta significación? Al regresar Vasari a Florencia, en junio de 1573, toda la decoración mural del Vaticano estuvo dirigida por un conciudadano del Papa, el hábil artista Lorenzo Sabatini (44). ¿Habría él consentido que una de las paredes más apreciadas del palacio papal - y de todo el mundo cristiano - fuese encomendada a un joven desconocido y a un pintor flamenco secundario? (45).

(41) G. Argote de Molina, Nobleza de Andalucia,ZSevilla, 1588, 188v; Fr. A. de la Calancha, Crónica moralizada del Orden de San Agustín en el Perú, Barcelona, 1638, 248, G. Baglione, opCcit., 32, ,77.VVerSO"

(42) A. Taja, loc. cit., debió basar su información en Baglione y en la identificación del emblema de los Buoncompagni (véase la nota 43). Esta versión se ha repetido frecuentemente desde entonces, véase, por ejemplo, L. Lanzi, op. cit., 195-196; A. Molfino, loc. cit.

(43) La escena está apretada en la tarja en forma de corazón del frente del sarcófago. Casi una tercera parte de la cartela está tapada por lo pierna de un demonio en huida, y en el espacio sobrante hay un vigoroso Moisés que sujeta una vara convertida en serpiente mientras escucha a Dios que aparece entre las llamas. El dragón de los Buoncompagni está representado en el margen izquierdo, con la intención probable de hacerlo aparecer como una extensión de la vara; véase nota 65 .

(44) C. van Mander, op. cit., $192 \mathrm{f}$, informa que Lorenzo estuvo a cargo de las pinturas en el Vaticano y que tuvo a muchos artistas jóvenes trabajando para él.

(45) Hasta esa época sólo se sabe que Pérez de Alesio trabajó como ayu. dante de Cesare Nebbia en la Villa d’Este. Véase D. Coffin, The Villa d'Estc at Tivoli, Princeton, 1960, 47,61. Mientras los pintores flamencos eran apreciados sobre todo por su habilidad en los paisajes y por su paciencia para diseñar groteschi. "Los Italianos piensan que sólo pora estos trabajos somos buenos (paisajes y groteschi) y que ellos lo son para los personajes", escribió C. van Mander, op. cit., I, 46. Van den Broeck trató de destruir ese prejuicio. Sin embargo, eso no lo convirtió en el candidato idóneo para la obra de la Capilla Sixtina. H. Noe, Loc. cit. 
Si fuera así, ¿no se liabría citado en las antiguas relaciones de las obras cumplidas por el Papa la renovación de los frescos en la Capilla Sistina? (46). Sin embargo, en ninguna de ellas se la menciona, aunque se hace referencia a la decoración mural emprendida por Gregerio en otros ambientes del Vaticano.

Un exanien más detenido de la cronología de los acontecinuientos, podrá proporcionar una explicación alternativa del origen de los frescos y evitar así las contradicciones mencionadas más arriba.

El terminws post quem para el inicio de ambos murales es establecido por el comienzo de las labores de De líano y Carnevale en la restauración de los frescos de Miguel Angel, entre octubre y diciembre de 1569 (47). Ninguna fecha anterior a ese año es aceptable. si se considera que la Capilla estuvo desafectada desde octubre de $1565(48)$.

Por otro laclo, la biografía de Pérez de Alesio proporciona evidencia interna para establecer un terminus ante quem anterior al que se ha reconocido hasta alıora. Nlesio fue admitido en la Academia de San Iucas de Roma en noyiembre de 1573 (49). Parn ser inscrito en esa Academia, un artista debía haber cumplido alguna obra pública de significación y haber alcanzado una edad respetable (50). Nacido alrededor de 1547 , en aquella fecha, Alesio había recientemente llegado a la edad recomendable de 25 anos. Al mismo tiempo, de las tres obras murales de importancia que dejó en Roma, sólo el fresco del

(46) Por ejemplo la rclocićn conservodo en el Archivo Buoncompogni o la que estableció Baglione, véose L. von Pastor, op. cit., IX, 916; G. Baglione, op. cit., Ed. Roma, 1935, 4-6.

(47) Véase las notos 27 y 28.

(48) Ninguno delies pinfores estuvo en Roma antes de eso fecha. Van den Eroeck aparece registrado en Romo desde 1565 en odelonte, cuando estuvo inscrito en la hermondad de Santa Moria in Compo Santo, véase G. J. Hoogewerft, loc. cit. Alesio estuvo ahi desde 1568: un dibujo de lo Biblioteca Pierpont Morçon, que se cree sea su retrato, tiene la inscripción: "Roma nel 1568 ", Y el primer pogo que recibió en Tivoli es de junio del mismo año. W. Heil,' "Palma Giovone als Zeichner", Jahrbuch d.Preusz. Kunstsammlungen, XLVII, 1926,05, fig. 8; H. Tietze, E. Tietze-Conrat, The drawings of the Venetion peinters in the 15th and 16th centuries, Nuevo York, 1944, 194, 216, No. 1045; D. Coifin, loc., eit.

(49) Libra di Introiti 1535-1633, II, f, 64. Archivo Storico della Accademia di Son Luea.

(50) 25 años ero la edad minimo aceptable para la matricula. En la Bula de Gragorio XIII (15 de diciembre de 1577 ) pora la reforma de la Accacemic, oporece específicomente mencionado el asunto de los ortistas jóvenes en términos que podríon referirse al caso de lo Capillo Sixtina: "... e perche ancora príncipiate c scolari senza niuna lunga practica... tirati della necesita, o dal'avidila del guadogno pigliano sopro di se l'incarico d'eseguire grondi lavori, onde poi questi riuscivano spogli e monconti di quelle perfezione tho alle buone Arti si cddice". En los Estatutos de G. de Vecchi (1596) se establce que el Principe debe taner al manos 30 oños, véose $M$. Missirini, Memcrie per cervire alla storia della Romana Acendemia di Son Luca, Roma, 1823, 196., 70; D. Martinez de la Peña, "artistos españoles en la Acodemio de Sán Lucas", Archivo Espoñol del Arsn, XLI, 1968, No. 164, 294-295. 


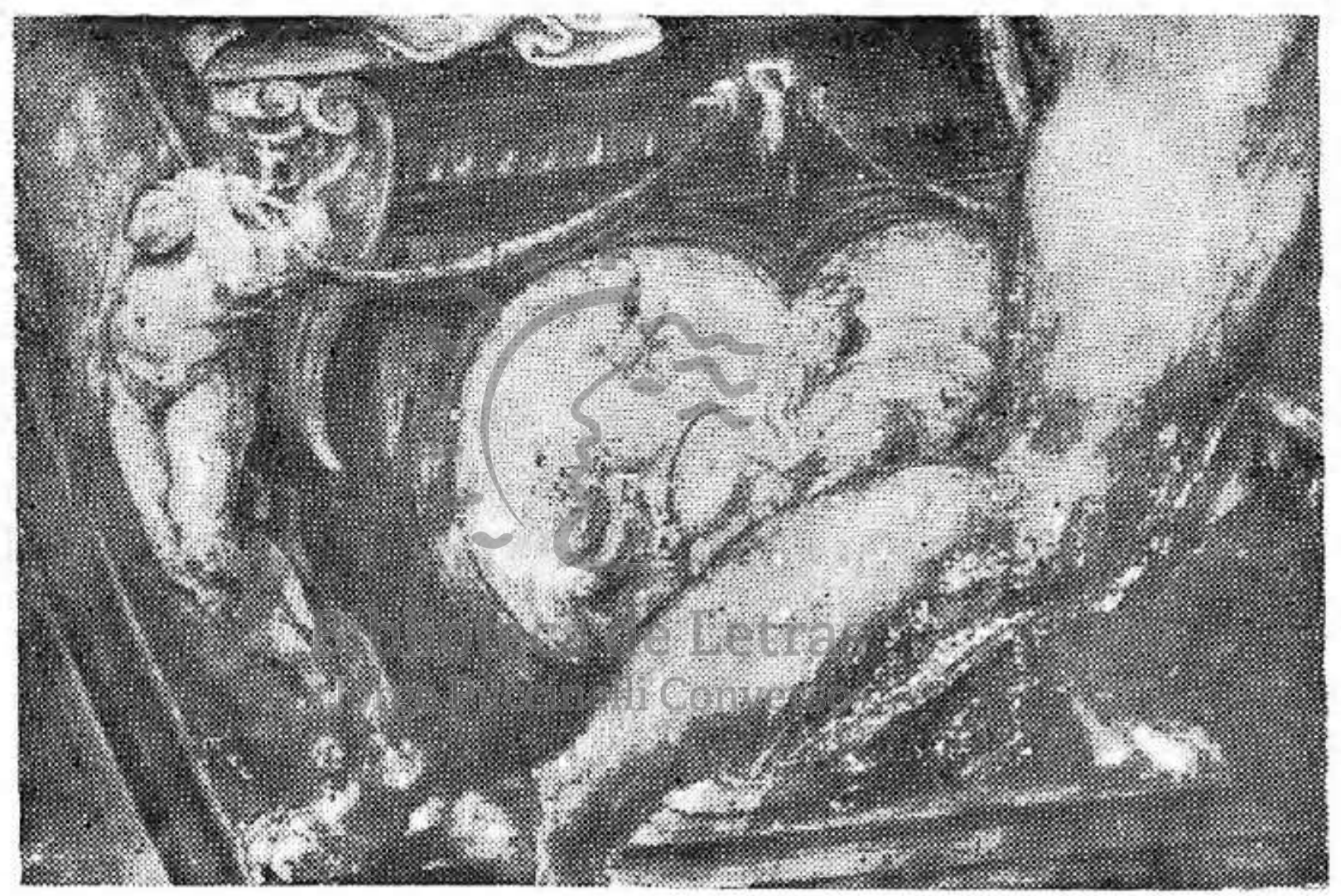

ROMA. "La defenso del cuerpo de Moisés". Salviati Capilla Sistina. (Detalle). 


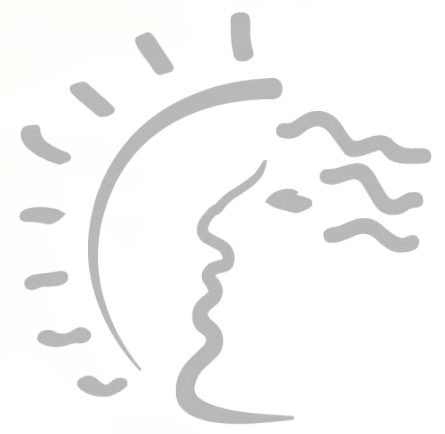

Biblioteca de Letras "Jorge Puccinelli Converso» 
Vaticano puede fecharse antes de 1573; las otras dos, las pinturas en el Oratorio del Gonfalone y las de la iglesia de Sant'Eligio dei Orefici, pertenecen a períodos más tardíos (51). De modo que para cumplir con ambas condiciones, habría sido necesario que Alesio hubiese completado el trabajo en la Capilla Sistina antes de noviembre de 1573.

El lapso entre diciembre de 1569 y noviembre de 1573, puede re. ducirse aún más, si se toma en cuenta que Giorgio Vasari fue llamado dos veces durante ese período para pintar en el Vaticano. Es difícil de creer, conociendo la admiración de Vasari por Miguel Angel y la importancia que le atribuía al Giudizio, que hubiera permanecido indiferente ante el hecho de que dos artistas desconocidos trabajasen en la sistina. Su primera visita tuvo lugar entre diciembre de 1570 y junio de 1571, cuando colaboró con Zucchi en la Torre Pia. La segunda vez fue llamado por Gregorio XIII, entre Noviembre de 1572 y Junio de 1573, para concluir la Sala Regia (52). Dado que Henri Van den Broeck aparece documentado como uno de los doce artistas que ayudaron a Vasari en esa empresa, puede asumirse que durante aquellos meses no estuvo comprometido simultáneamente en la Capilla papal en calidad de maestro independiente (53).

Recapitulando, quedan tres nomentos antes de Noviembre de 1573 en los cuales Alesio y Van den Broeck pudieron haber llevado a cabo sus obras: 1) el período de cuatro meses entre Junio y Noviembre $1573 ; 3$ ) el año de Dicicmbre de 1569 a Diciembre de 1570 .

(51) La obra en el Gonfalone está documentada entre 1575-76; véase K. Oberhuber, "Jacop Bertoia in Oratorium von S. Lucia del Gonfalone in Rom" Römische Historische Mitteilungen, 1958-1959, Colonia, 1960, 253-254, No. 44. Y de los documentos estudiados poradottoressa $M$. Qittoria Brugnoli Pace, se desprende que el fresco de la pared del altar de Sant'Eligio dei Orefici no fue pintado en el período" del 1566-1576. La iglesia esfa" mencionada en el: Anonimo Spagnolo, f. 104. Cod. Chigiano I, V, 167, Biblioteca Vaticana. Por lo tanto debió realizarse durante la segunda estadía de Alesio en Roma, en 1581-83. Esta fecha es confirmada por el hecho de que Van Mander, quien dio una cuenta cabal de los trabajos de Alesio antes de 1576, no mencionó a Sant'Eligio. Agradezco sinceramente a la Dottoressa $M$. V. Brugnoli Pace por haberme informado amablemente sobre los resultados de su investigación documentaria. Véase también M. V. Brugnoli, "Su Raffaello Architetto, la Capella Chisi e la Chiesa di Sant'Eligio", Rivista' dell'Istituto Nazionale d'Archelogia e Storia dell'Arte, XVI, 1969.

(52) Los pagos por el trabajo en las tres Capillas de Pío $V$ están documentados en Libri dai Conti delle Deposit. Gen. della Camera Apostolica, 1570 1572, véase Vasari, Milanesi, op. cit., VII, 715; A. Taja, op. cit., 951: G. Moroni, Dizionario di erudizione storico-ecclesiastica, Venecia, 1840-1861, IX, $156 \mathrm{f}$. Para la participación de Vasari en la Sala Regia, véase los documentos citados por M. Vaes, op. cit., 217 , n. 3; A. Venturi Śtoria dell'arte italiana. IX.'La Pittura del Cinquecento, Milán, 1925-1934, 6, $304 \mathrm{f}$.

(53) Van Mander, op. cit., 184v, creía que Van den Broeck trabajó con Pieter de Witte como ayudante de Vasari en la Sala Regia. Esto está confirmado por una carta de Vasari a V. Borgini (3 de feb. de 1573), véase G. Gaye, Carteggio inedito d'Artisti dei secoli XIV, XV, XVI, Florencia, 1839-40, II, 361 . Es extraño que el fresco de la Resurrección de Van den Broeck, siendo obra de un Flamenco, haya escapado de la memoria de Van Mander y que no la mencionara en su libro. 
El primer lapso es no sólo muy corto para semejante tarea, sino que coincide, como ya se mencionó, con la época cuando Sabatini fue el "pintor papal" que tenía a su cargo la decoración del Palacio del Vaticano (54). Su nombre está asociado con todos los frescos pintados en la época y ciertamente no habría sido dejado de lado al emprenderse obras en el ambiente más importante del Palacio. Así que parece poco probable que los murales hubiesen sido renovados durante aquellos meses del año 1573 (55).

El período que se extiende desde diciembre de 1569 a diciembre de 1570 , habría parecido a primera vista la época más indicada para la participación de Alesio y Van den Broeck; pero no puede ser tomatdo seriamente en consideración, porque no existiría ningún motivo paira la incorporación del emblema de los Buoncompagni.

Es mucho más aceptable considerar el trabajo de Alesio y de Van den Broeck en la Capilla como la etapa final del proceso de restauración conducido por el Cardenal Rusticucci. Varios factores señalan en ese sentido. Primeramente, la actividad de Pérez de. Alesio y Van den Broeck en Roma no se limitó a la época del Papa Gregorio, como se implica de los textos de Van Mander y Baglione, sino que empezó anteriormente durante el pontificado de Pío $V$, e incluso antes, desde 1565, en el caso del artista flamenco (56). Alesio estuvo en 'Tívoli en 1568-69 y en los años siguientes tuvo tratos con dos dignatarios de la época de Pío V: el Cardenal Rambouillet y significativamente, con Monsignor Fantino Petrignahi (57), quien entre 1564 y 1576 está documentado como "Prefetto del Palazzo Apostolico" (58).

(54) C. van Mander, op. cit., $192 f$

(55) La impresión que uno puede recibir det texto de Van Mander de que Alesio y Guidonio pertenecieron al grupo de pintores que trabajaron a las órdenes de Sabatini, es engañosa. La colaboración con Sabatini se menciona especificamente en los casos de Raffaellino Motta y Ricardo Sazzi, cuyos trabajos se describen junto a los de Alesio y Guidonio; sin embargo el texto sobre este último no menciona ninguna relación con Lorenzo Sabatini, simplemente dice: "Noch isser geweest te Room in mijnen tijd...". En otras palabras cuando Van Mander llegó a Roma, en junio de 1574, encontró a Sabatini a cargo de las pinturos del Vaticano.. Pero los frescos de la Capilla Sixtina estaban listos ya en esa fecha y pudieron haber sido pintados mucho antes de la llegada del artista Emiliano.

(56) G. Mancini, op. cit., I, 222, afirma correctamente la presencia de Alesio en tiempos de Pío V; C. van Mander, loc. cit.; G. Bagliones, op. cit., 32, 77; véase la nota 48 .

(57) Charles d'Angennes de Rambouillet (1530-1587), era llamado Cardenal Ramboglietti y es el misterioso Eonfiglietti que se menciona en las anotaciones de Bellori a Baglione. Era obispo de Le Mans y fue trasladado a Roma, donde Pío $V$ lo designó cardenal en mayo de 1570. Pérez de Alesio le fue presentado por un cierto Salvador de Venecia y viajó con él a Viterbo y Tarquinia. G. Baglione, op. cit., (Roma, 1935), 31 ; véase también D. Mahon, Studies in Seicento ort and theory, Londres, 1947, 319, n. 122; L. von Pastor, op. eit., VIII, 119-120.

Fantino Petrignani $(+1600)$ trabajó para Pio $V$ y Gregorio XIII. Como Prefetto del Palacio Vaticano debía ocuparse de su conservación y decoración. En 1578 fue enviado en calidad de Nuncio a Nópoles y luego a España. Esta afinidad con el mundo Ibérico explica su relación con Pérez de Alesio, quien era 
Rambouillet fue nombrado cardenal por Pío $V$ en 1570 y Alesio debió de haber trabajado para él por algún tiempo desde entonces. Por consiguiente, ya sea Monsignore Petrignani o el Cardenal Rambouillet, habiendo estado satisfechos con los servicios de Alesio, pudieron haber recomendado al joven artista a Rusticucci cuando éste debió restaurar los frescos de la pared dañada de la Capilla papal.

El pontificado de Pío $V$ es suficientemente conocido por haber sido una época dominada totalmente por consideraciones ascéticas $y$ místicas, para que sea comprensible que el interés del Papa por la Capilla Sistina era exclusivamente religioso (59). La Capilla ya no era vista como un templo del arte, sino tan sólo como el lugar sagrado reservado para que el Papa oficie el culto divino y cuya decoración era concebida tan solo Ad Maiorem Dci Gloriam. El hecho de que el arreglo de la Capilla hubiese sido encomendada a un Cardenal que era conocido por tener "piu prudenza che doctrina" y por ser "di moto tardo, ma diligente, (e) di buoni sentimenti..." (60) cuadra perfectamente con la atmósfera que vivían la Iglesia y la sociedad romana de la época. Los días cuando Pablo III había ido personalmente al taller de Miguel Angel para solicitarle que aceptara un pedido, estaban muy lejos. La actitud de San Pío era de indiferencia y de economía hacia el arte. Pintores jóvenes y marginales fueron llamados para retocar la Capilla Sistina (61). Las artes eran apreciadas tan solo en cuanto servían de vehículos al dogma, y sus dimensiones creativas y

de origen español. Véase G. Mancini, op, cit., I, 233; J. Hess, Kunstgeschichte Studien zum Renaissance und Barock, Roma, 1967, T, 23; G. Moroni, op. cit., $X L I, 260, \mathrm{CII}, 361$.

(58) Como una prúbalmás decla lfamiliaridadede Alesio con Pio $V$ aparece un retrato del Papa en una nómina de los cuadros dejados por el artista a su muerte, en Lima, véase R. Porras Barrenechea, "Un inventario iconográfico del Siglo XVI. El pintor Mateo de Alesio"; Revista Cultura Peruana, 90, Lima, 1955. El retrato debió ser encargado por la Universidad de San Marcos de Lima, la que fundada en mayo de 1551 fue reconocida por Pio $V$ en una Bula del 25 de julio de 1571 .

(59) La primera preocupación de Pio $V$ fue cubrir los desnudos dejados por Daniele da Volterra en el Giudizio. El Papa reaccionó vigorosamente contra la desnudez de muchas estatuas clásicas. En general su comprensión del arte fue en extremo limitada, debido a sus prejuicios hacia el cuerpo humano y a su austeridad excesiva en materia económica. Véase la nota 61 y $\mathrm{L}$. von Pastor, op. cit., VII, $81-86$.

(60) Girolamo Rusticucci (1537-1603) fue secretario de Antonio M. Ghislieri durante nueve años, antes de que éste fuera el Papa Pio $V$. En un manuscrito de 1599 aparece descrito como "d'ingegno posato, ma sagace", véase G. Moroni, op. cit., II, 783, No. 6.

(61) De manera parecida, en tiempos de Pio $V$ los frescos de la capilla de Santi Martino e Sebastiano (1567-68), en el Vaticano, fueron encargados a Giulio Mazzonni, un ayudante de Vasari más conocido como stuccatore que como pintor. Una carta de Vasari escrita en Ruma en marzo de 1567, describe la austeridad y la miseria en la que Roma habia caido y la "mezquindad en el vivir" que se habia extendido desde el Vaticano a toda la Ciudad Santa; citads. por S. J. Freedberg, op. cit., 294; véase G. P. Chattard, op. cit., III, 334. 
expresivas eran pasadas por alto. Estos fueron los tiempos cuando Pío V mandó hacer una copia del Juicio Final de Fra Angelico atraido exclusivamente por la leyenda de santidad creada en torno a la personalidad del pintor (62). Y en esta época, probablemente por la segunda mitad de 1571, Arrigo Fiammingo y Matteo da Jeece fucron llamados para una tarea que las autoridades del Vaticano deben haber visualizado simplemente como una manera económica de repetir dos frescos dañados de otros tiempos (63).

Durante el mes de mayo de 1572 , mientras el trabajo estuvo probablemente en proceso, Pío V falleció y Gregorio XIII fue elegido. En esas circunstancias, Alesio sin duda agregó el dragón de los Buoncompagni como un sutil homenaje dedicado al nuevo Papa. El animal heráldico es pequeño y apenas perceptible, a pesar de encontrarse en un lugar tan central. Por añadidura parece que estuviera fuera de contexto en la escena de la Zarza Ardiente (64). No obstante, su presencia puede ser interpretada como una transformación extrema de la vara de Moisés, la cual, convertida en serpiente como testimonio de la visión divina en la narración bíblica, asume aquí el aspecto de un dragón en uno de sus extremos (65). El blasón del Papa es, de ese modo, asimilado a un origen sobrenatural, el cual irradia un mandato casi divino sobre la investidura recién recibida por Gregorio al relacionar al nuevo Papa por filiación emblemática (66) con aquél que fuera considerado como el San Pedro de la Antigua Ley (67).

El encargo de Rusticucci en la Capilla Sistina debió haber sido concluido antes de noviembre de 1572 , cuando aquél abandonó Roma para radicarse en Sinigaglia (68). La fecha coincide exactamente con Biblioteca de Letras

\section{«Jorge Puccinelli Converso»}

(62) Ver L. von Pastor, op. cit., VIII, 81f; F. Zeri, op. cit., 61, fig. 50 , para la copia de Fra Angelico, que curiosamente fue piritada por B. Spranger, uno de los artistas más sensuales entre los manieristas nórdicos.

(63) Sintomáticamente, G. Celio, loc. cit., cuando escribió sobre los dos murales del siglo XVI, dijo: "ma con volerle rinfrescare, non sono piu quelle".

(64) Cuando Gregorio XIll tenía motivos para colocar su emblema lo hacía en una forma menos oculta; por ejemplo, en una de las habitaciones de sus apartamentos privados. C. van Mander, op. cit., 193, lo describe como una obra de Raffaellino da Reggio.

(65) La contradicción entre el hecho de que el dragón no debia tener cola y de que Moisés aparentemente lo sostiene por ella, se resolvió escondiendo el punto de contacto entre ambos detrás de una de las piernas del demonio.

(66) Moisés fue el prototipo tanto de Cristo como de San Pedro, y su vara estuvo guardada en San Giovanni in Laterano junto con las reliquias más preciosas del propio San Pedro, véase L. Réau, Iconographie de I'art chretien, París, $1965,11,176$.

relación.

(67) Quiero agradecer a la Dra. Jennifer Montagu por señalar esta posible

(68) G. Morcni, op. cit., LXVI, 259. Es normal que habiendo sido duran. te quince años un intimo colaborador te Pío $V$, dejara su posición en el Vaticano después de la elección del nuevo Papa que tuvo lugar el 14 de mayo de 1572. La demora de su partida pudo deberse al hecho de que no había concluido la tarea en la Capilla Sixtina. 
la llegada de Vasari para la decoración de la Sala Regia y la incorporación subsecuente de Henry Van den Broeck entre sus ayudantes (69). Ese suceso está ciertamente más en conformidad con el tratamiento que se solía otorgar a los artistas flamencos en Roma en la época, y muestra cómo las cosas volvieron a su cause normal una vez que la extrema austeridad del Papa anterior fue dejada atrás (70).

Así, han podido explicarse los principales hechos relacionados a los dos murales àquí estudiados. La cuestión de la fecha en sí, vale decir si las pinturas se originaron un año o dos antes de lo que se ha asumido habitualmente, puede ser un asunto de interés puramente académico. Pero si los años de diferencia establecidos son los que separan al pontificado del ascético San l'ío de la renovada actividad artística de los tiempos de Gregorio XIII, el asunto tendrá mayor significación para alcanzar un entendimiento más cabal de los frescos y para explicar la presencia de éstos en la Capilla Sistina. Un artista cono Pérez de Alesio fue muy conscientu de esa diferencia, y siempre procuró asociar su nombre con el del Papa que se comportó como un genuino mecenas y que volvió a la tradición de la iglesia de patrocinar a las artes con generosidad y visión.

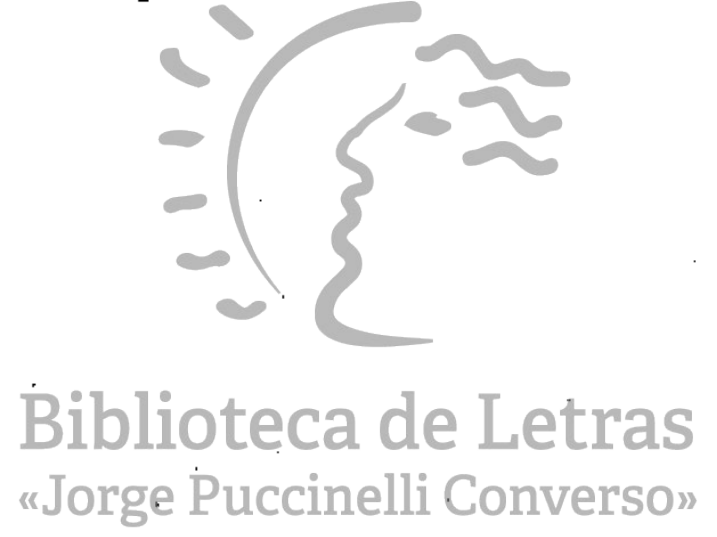

(69) Véase la nota 53.

(70) Un argumento adicional para apoyar esta datación la proporciona la anécdoto narroda por Mancini acerca de El Greco, quien está documentodo én la Accademia di San Luca, en Roma, en setiembre de 1572. En la época en que el Papa Pio estuva haciendo cubrir algunos desnudos del Giudizio de Miguel Angel -informo Mancini- El Greco exclamó, 'se si buttase a terra tutta i'opera, l'haverebbe fatta con honesta et decenza non inferiore a quella di bonta di pittura", y habiendo provocado así la enemistad de los pintores romanos tuvo que viajar a España. Usualmente se ocepta que El Greco dejó Roma en 1573 (pora ir a. Venecia); y si hay algo de cierto en lo afirmoción de Mancini, con. firmario que hubo obras en proceso en la Capilla Sixtina durante el año 1572 . G. Mancini, op. cit., I; 230-1; D. Martínez de lo Peña, "El Greeo en lo Academia de Són Lucos", 'Archivo Español de Arte, XL, 1967, 97. 